\title{
Effects of release modifier on Carvedilol release from Kollidon SR based matrix
}

\author{
Urmi Das, *Mohammad Salim Hossain \\ Department of Pharmacy, Noakhali Science and Technology University, Sonapur, Noakhali-3814, Bangladesh
}

\begin{abstract}
Sustained release Carvedilol matrix tablets constituting Kollidon SR were developed in this study in an attempt to investigate the effect of release modifiers on the release profile of Carvedilol from matrix. Three matrix tablet formulations were prepared by direct compression of Kollidon SR in combination with release modifier (HPMC and Microcrystalline Cellulose) and magnesium stearate. Tablets containing only Kollidon SR with the active ingredient demonstrated a rapid rate of drug release. Incorporation of HPMC in the matrix tablet prolonged the release of drug but incorporation of Microcrystalline Cellulose showed superimposable release pattern with an initial burst effect as confirmed by mean dissolution time and Higuchi release rate data. After 7 hours of dissolution, Carvedilol release from the matrix systems were 91.42\%, 83.41\%, from formulation F1 and F2 respectively. Formulation F3 exhibited $100 \%$ release at 4 hours. All the tablet formulations showed acceptable pharmaco-technical properties and complied with the in-house specifications for tablet weight variation, friability, hardness, thickness, and diameter. Prepared tablets also showed sustained release property for carvedilol. The drug release mechanism from the matrix tablets of F1 and F2 was found to be followed by Fickian and F3 by Non-Fickian mechanism.
\end{abstract}

Key Words: Microcrystalline cellulose, Carvedilol, sustain release, HPMC, swelling study, Fickian release.

\section{INTRODUCTION}

The ultimate goal of any drug delivery system is effective disease or disorder management, minimum side effects and greater patient compliance in a cost effective manner. The drug therapeutic indices could be maximized while indices of adverse reactions or side effects could be minimized by regulating the drug release in body in a welldefined controlled manner. Most conventional dosage forms e.g., solution, suspension, capsule, tablet etc function merely to place a drug at the site of administration and pay no regard to the regulation of release and absorption or the duration and targeting of drug in the body. If any attempt is made to maintain drug blood level in the therapeutic range for longer periods by, for example increasing the dose, toxic levels may be produced at any time

\footnotetext{
*Corresponding Author:

Mohammad Salim Hossain

Assistant Professor, Department of Pharmacy

Noakhali Science and Technology University

Sonapur, Noakhali-3814, Bangladesh

E-mail: pharmasalim@yahoo.com

Contact No.: +88-01711-200410
}

(Beringer et al., 2005). Therapeutic efficacy \& safety of drugs administered by conventional methods can be improved by precise spatial and temporal placement within the body, thereby reducing both the size and number of doses.

In recent years considerable attention has been focused on the development of new drug delivery systems. In spite of the recent technological advances in the fabrication of oral controlled-release dosage forms, particular attention has been paid to the regulation of drug release rate by means of monolithic devices, whereby prior dispersion of the drug in a polymer matrix is carried out (Cardinal, 1984). Embedding a drug within an insoluble matrix provides a convenient means of controlling the drug release. In such a system, drug release is preceded by penetration of the dissolution medium into the porous matrix to dissolve the drug, followed by diffusion or leaching of the dissolved molecules out of the matrix. Solid drug on the matrix surface will be dissolved and released first. Upon exhaustion of the surface drug, the depletion zone will then 
Table 1: Composition of Carvedilol SR matrix tablets.

\begin{tabular}{lccc}
\hline Ingredients (mg) & \multicolumn{3}{c}{ Formulation Code } \\
\cline { 2 - 4 } & F1 & F2 & F3 \\
\hline Carvedilol & 80 & 80 & 80 \\
Kollidon SR & 300 & 200 & 200 \\
Hydroxypropylmethylcellulose & - & 100 & - \\
Microcrystalline Cellulose (Avicel) & - & - & 100 \\
Magnesium stearate & 3 & 3 & 3 \\
Total weight & 383 & 383 & 383 \\
\hline
\end{tabular}

increased progressively as the solid drug front recedes into the matrix (Billah et al., 1998).

A wide array of polymers has been employed as drugretarding agents each of which presents a different approach to the matrix concept. Plastic matrix systems, due to their chemical inertness and drug embedding ability, have been widely used for sustaining the release of drug. Liquid penetration into the matrix is the rate-limiting step in such systems unless channeling agents are used (Reja et al., 2003).

In the present study, the effect of release modifiers on the release profile of Carvedilol from Kollidon SR based matrix has been investigated. Matrix tablets each containing $80 \mathrm{mg}$ of Carvedilol were formulated employing HPMC and Microcrystalline Cellulose as release modifier. The effects of these two release modifiers and processing condition on drug dissolution were evaluated. The release mechanism of drug from the sustained release dosage form were explored and explained with the help of exponential model.

\section{MATERIALS AND METHODS}

The active pharmaceutical ingredient, Carvedilol was received as generous gift from Drug International Ltd. Kollidon SR (blend of $80 \%$ polyvinyl acetate and $19 \%$ povidone) was obtained from BASF Bangladesh Limited. Microcrystalline cellulose
(Ming Tai Chemical Co. Limited, Taiwan), and Hydroxypropylmethylcellulose (Colorcon, USA) and Magnesium stearate (Colorcon, USA) were also received as from the respective source. Hydrochloric acid (Merck, Germany) and Potassium dihydrogen phosphate (Merck, Germany) were used as dissolution medium. All other chemicals used were of analytical grade.

\section{Preparation of Carvedilol Matrix Tablet}

The active ingredient, matrix polymer, release modifiers and lubricant were blended together and made into tablets by direct compression at a fixed compression force. Table 1 summarizes the formulation of carvedilol sustained release tablets. All the components required for tablets were blended for 10 minutes. Particular attention was given to ensure thorough mixing and phase homogenization. The appropriate amounts of the mixture were then compressed using a hydraulic press equipped with a $13 \mathrm{~mm}$ flat faced punch and die set. The compression force and compression time were 5 ton and 1 minute respectively. Before compression, the surfaces of the die and punch were lubricated with magnesium stearate. All the preparations were stored in airtight containers at room temperature for further study. However, this process of tablet manufacturing differs from practical condition to a larger extent and does not consider some critical tableting parameters such as porosity, tablet hardness and versatility of process conditions. Data generated from such systems require sufficient scaling up and should not be directly extrapolated to commercially prepared controlled release tablets. Moreover, different scientists described their techniques to the study on drug release behavior from matrix tablets.

\section{Physical Characterization of Matrix Tablets}

To evaluate physicochemical properties and the ability of the tablet to withstand mechanical han-

Table 2: Physical characterization of Carvedilol SR matrix tablets.

\begin{tabular}{cccccc}
\hline $\begin{array}{c}\text { Formulation } \\
\text { Code }\end{array}$ & $\begin{array}{c}\text { Friability (\%) } \\
\mathbf{n = 4}\end{array}$ & $\begin{array}{c}\text { Weight variation } \\
\mathbf{n = 1 0}\end{array}$ & $\begin{array}{c}\text { Hardness (kgf) } \\
\mathbf{n = 5}\end{array}$ & $\begin{array}{c}\text { Thickness (mm) } \\
\mathbf{n = 5}\end{array}$ & $\begin{array}{c}\text { Diameter (mm) } \\
\mathbf{n = 5}\end{array}$ \\
\hline F1 & $0.23 \pm 0.01$ & $382.94 \pm 0.21$ & $33.62 \pm 0.56$ & $3.00 \pm 0.03$ & $13.00 \pm 0.05$ \\
F2 & $0.23 \pm 0.01$ & $382.55 \pm 0.26$ & $23.47 \pm 0.13$ & $3.00 \pm 0.03$ & $13.04 \pm 0.05$ \\
F3 & $0.25 \pm 0.01$ & $382.79 \pm 0.26$ & $26.21 \pm 0.69$ & $3.00 \pm 0.02$ & $13.00 \pm 0.03$ \\
\hline
\end{tabular}

All values represent mean \pm SEM 
Table 3: Kinetic parameters of formulated Carvedilol SR matrix tablets.

\begin{tabular}{cccccccccccc}
\hline Formulation Code & \multicolumn{2}{c}{ Zero order } & \multicolumn{2}{c}{ First order } & \multicolumn{2}{c}{ Higuchi } & \multicolumn{2}{c}{ Korsmeyer-Peppas } & \multicolumn{2}{c}{ Hixson-Crowell } \\
\hline & $\mathbf{K}_{\mathbf{0}}$ & $\mathbf{R}^{\mathbf{2}}$ & $\mathbf{K}_{\mathbf{1}}$ & $\mathbf{R}^{\mathbf{2}}$ & $\mathbf{K}$ H & $\mathbf{R}^{\mathbf{2}}$ & $\mathbf{n}$ & $\mathbf{R}^{\mathbf{2}}$ & $\mathbf{K}_{\text {HC }}$ & $\mathbf{R}^{\mathbf{2}}$ \\
\cline { 2 - 12 } F1 & 16.39 & 0.64 & -0.34 & 0.82 & 31.43 & 0.83 & 0.28 & 0.88 & 0.31 & 0.31 \\
F2 & 9.70 & 0.70 & -0.25 & 0.85 & 31.78 & 0.89 & 0.39 & 0.92 & 0.31 & 0.39 \\
F3 & 9.25 & 0.60 & -1.75 & 0.99 & 46.77 & 0.87 & 0.50 & 0.93 & 0.35 & 0.35 \\
\hline
\end{tabular}

dling and transport, various parameters- friability, weight variation, hardness, thickness and diameter were to be measured.

\section{Drug Content Assay}

Drug content of the sample solution i.e. the quality of the drug release was determined by Spectrophotometric analysis and the absorbance measured at $276 \mathrm{~nm}$ by using Shimudzu UV spectrophotometer. In each time respective dissolution media was used as blank. For each value of absorbance, the concentration of the corresponding solution was calculated by using equation of the standard curve and then the amount of drug of each vessel was determined.

\section{Swelling Study}

The extent of swelling was measured in terms of percentage of diameter and thickness gained by the tablet. Two tablets from each formulation were exposed to $\mathrm{pH} 7.4$ phosphate buffer. At the end of 2 hour, tablets were withdrawn, soaked with tissue paper and the swelling behaviors of the formulations were observed.

\section{Surface pH Study}

The surface $\mathrm{pH}$ of the formulated tablets was determined in order to investigate the possibility of any side effects in vivo. As an acidic or alkaline $\mathrm{pH}$ may cause irritation to the gastric mucosa, it was determined to keep the surface $\mathrm{pH}$ as close to neutral as possible. The tablet was allowed to swell by keeping it in contact with $5 \mathrm{ml}$ buffer ( $\mathrm{pH}$ 7.4) for 1 hour at room temperature. The $\mathrm{pH}$ was measured by bringing the electrode in contact with the surface of the tablet and allowing it to equilibrate for 1 minute.

\section{Drug-Excipients Interaction Study}

Assessment of possible incompatibilities between an active drug substance and excipients forms an important part of the preformulation stage during the development of solid dosage form. Among the various methods, UV-spectroscopy is the simplest method for the evaluation of possible incompatibilities. The UV spectra of each the four formulations were compared with the standard spectrum of Carvedilol.

\section{In vitro Dissolution Study}

The in vitro release studies of carvedilol from the prepared matrix tablet were conducted by USP Dissolution tester (TDT-08L plus, Electrolab, India) type II (paddle method) at a constant temperature of $37 \pm 0.5{ }^{\circ} \mathrm{C}$ and $100 \mathrm{rpm}$ speed. The dissolution studies were carried out for 7 hours duration. As dissolution medium, $0.1 \mathrm{~N} \mathrm{HCl}(900 \mathrm{ml})$ was used for the first 2 hours and then after the $2^{\text {nd }}$ hour, the acid media was replaced by phosphate buffer $(\mathrm{pH}$ 7.4) for the next 5 hours. At predetermined time intervals, $5 \mathrm{ml}$ sample was withdrawn and replaced with fresh dissolution medium. After appropriate dilution the samples were analyzed by a double beam UV spectrophotometer at a wavelength of 241 $\mathrm{nm}$. Cumulative percentage of the drug released was calculated.

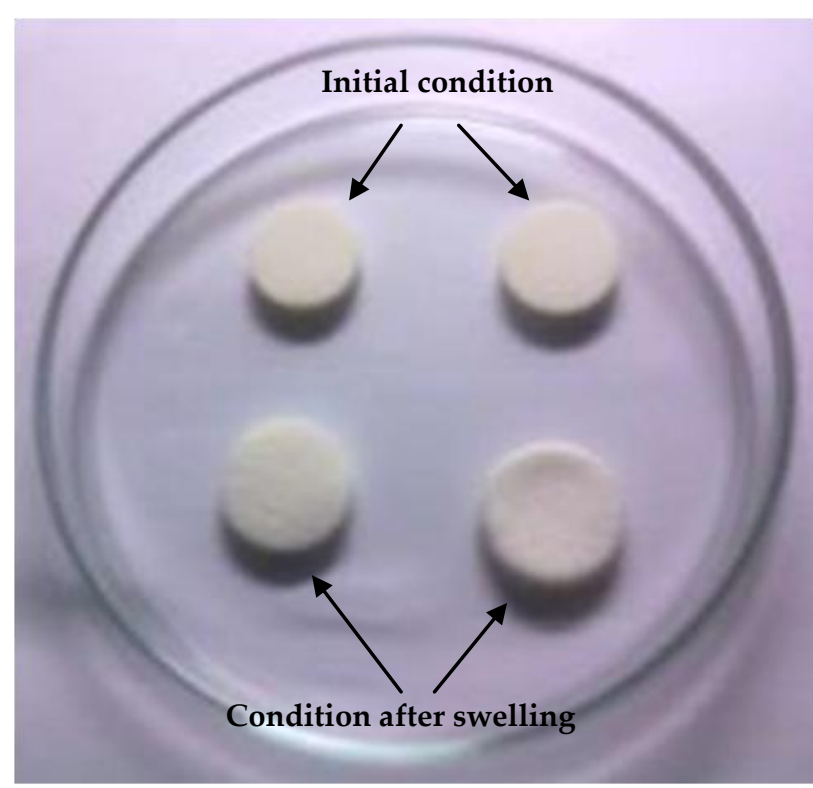

Figure 1: Photograph of normal and swelled tablets. 


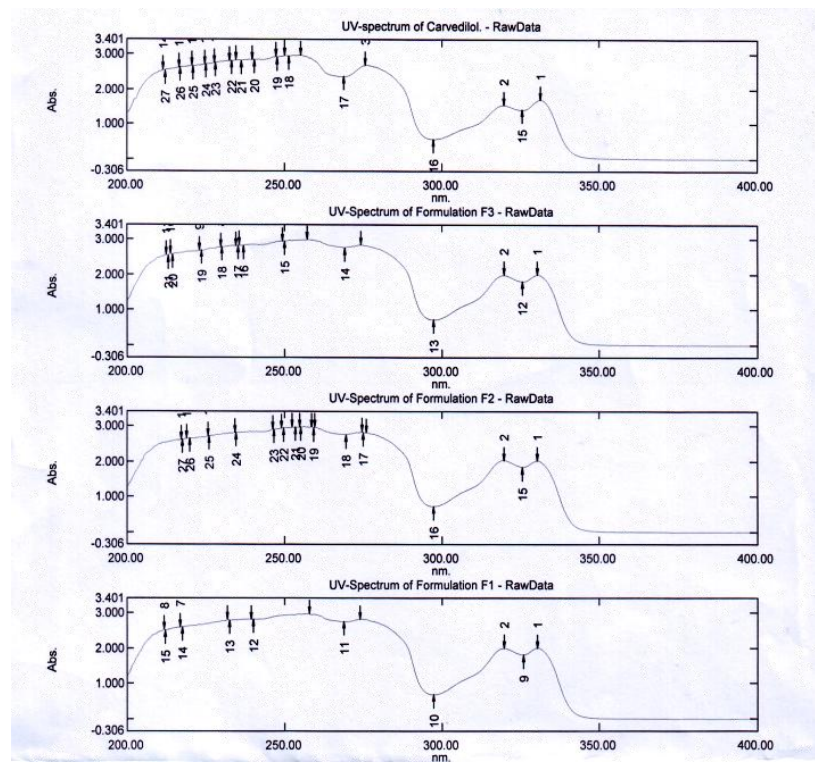

Figure 2: UV spectrum of pure drug (Carvedilol) and formulations.

\section{Characterization of Release Kinetics}

In order to study the exact mechanism of drug release from the matrix tablets different kinetic equations were applied. The release data were fitted to the following equations:

Zero-order equation: $Q=K_{0} t$

Where, $Q$ is the amount of drug released at time $t$, and $\mathrm{k}_{0}$ is the release rate.

First-order equation: $\log Q_{t}=\log Q_{0}+K_{1} t / 2.303$

Where, $Q$ is the initial amount of drug, $Q_{t}$ is the percent of drug release at time $t$, and $k_{1}$ is the release rate constant.

Higuchi's equation (Higuchi, 1961): $Q=K_{H} t^{1 / 2}$

Where, $Q$ is the percent of drug release at time $t$, and $\mathrm{K}_{\mathrm{H}}$ is the diffusion rate constant.

These models fail to explain drug release mechanism due to swelling (upon hydration) along with gradual erosion of the matrix. Therefore, the dissolution data was also fitted to the well-known exponential equation (Korsmeyer-Peppas equation), which is often used to describe the drug release behavior from polymeric systems (Ritger and Peppas, 1987; Korsmeyer et al., 1983).

Korsmeyer-Peppas equation: $\log \left(M_{t} \mid M_{f}\right)=\log k+n \log t$ Where, $M_{t}$ is the amount of drug release at time $t, M_{f}$ is the amount of drug release after infinite time; $\mathrm{k}$ is a release rate constant incorporating structural and geometric characteristics of the tablet, and $\mathrm{n}$ is the diffusional exponent indicative of the mechanism of drug release.

To clarify the release exponent for different formulations of matrix tablet, the log value of percentage drug dissolved was plotted against log time for each formulation. A value of $\mathrm{n}=0.45$ indicates Fickian (case I) release; $>0.45$ but $<0.89$ for Non-Fickian (anomalous) release; and $>0.89$ indicates super case II type of release. Case II generally refers to the erosion of the polymeric chain and anomalous transport (Non-Fickian) refers to a combination of both diffusion and erosion controlled-drug release (Shato et al., 1997).

Hixson - Crowell release equation describes the drug releases by dissolution and with the changes in surface area and diameter of the particles or tablets.

Hixson-Crowell release equation: $\sqrt[3]{Q_{0}}+\sqrt{Q_{t}}=K_{H C} t$

Where, $Q_{0}$ is the initial amount of drug, $Q_{t}$ is cumulative amount of drug release at time $t, K_{H C}$ is a Hixson-Crowell release constant and $t$ is time in hours.

Mean dissolution time (MDT) was calculated from dissolution data using the following equation (Mockel and Lippold, 1993) -

$M D T=(n / n+1) k^{-1 / n}$

Where, $\mathrm{n}=$ release exponent and $\mathrm{k}=$ release rate constant.

A higher value of MDT indicates a higher drug retaining ability of the polymer and vice-versa.

\section{Statistical Analysis}

To justify the statistical significance, student " $\mathrm{t}$ " test was carried out and $p$ value less than 0.05 (at 5\% confidence level) was considered as statistically significance.

\section{RESULTS AND DISCUSSION}

All the tablet formulations showed acceptable pharmacotechnical properties and complied with the in-house specifications for tablet weight variation, friability, hardness, thickness, and diameter (Table 2). Tablet thickness was within the range of $2.9 \mathrm{~mm}$ to $3.1 \mathrm{~mm}$, diameter was within the range of $13 \mathrm{~mm}$ to $13.2 \mathrm{~mm}$. The percentage friability for all 


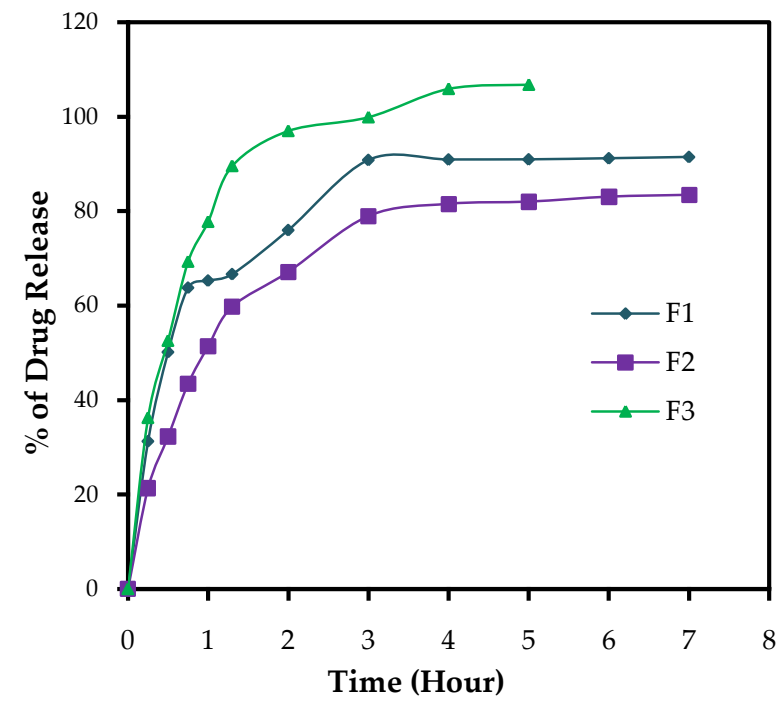

Figure 3: Zero order plot of release kinetics of Carvedilol SR tablets of different formulations.

tablets was below $1 \%$, indicating that the friability was within the official limits. Tablet hardness was found to be good depending on the compression force applied. Weight variation and values was also within acceptable range. Swelling behaviors of the formulated Carvedilol SR were observed. Polymers, used in the formulations-Kollidon SR, HPMC, and Microcrystalline Cellulose showed different degree of swelling ability (figure 1). The diameter and thickness of the tablets of formulation F1, F2, F3 were increased up to $101.5 \%, 107.69 \%, 103.85 \%$ and $108.33 \%$, 133.33\%, 116.66\% respectively. Carvedilol SR tablets of all the formulations showed surface $\mathrm{pH}$ values close to the neutral value- $\mathrm{pH} 6.25$ for formulation F1, $\mathrm{pH} 6.56$ for $\mathrm{F} 2$ and $\mathrm{pH} 6.36$ for F3, which indicates no risk of mucosal damage or irritation. The UV spectral analysis showed that there was no appearance or disappearance of any characteristic peak. In spectra, formulations shows same absorption pattern as that of pure drug, which confirms the absence of chemical interaction between drug and excipients. The spectra are shown in the figure 2 .

\section{In vitro Dissolution Studies of the Formulated Tablets}

In the present study, an attempt has been taken to develop sustained release tablets of Carvedilol by direct compression method using Kollidon SR in combination with release modifiers. HPMC and

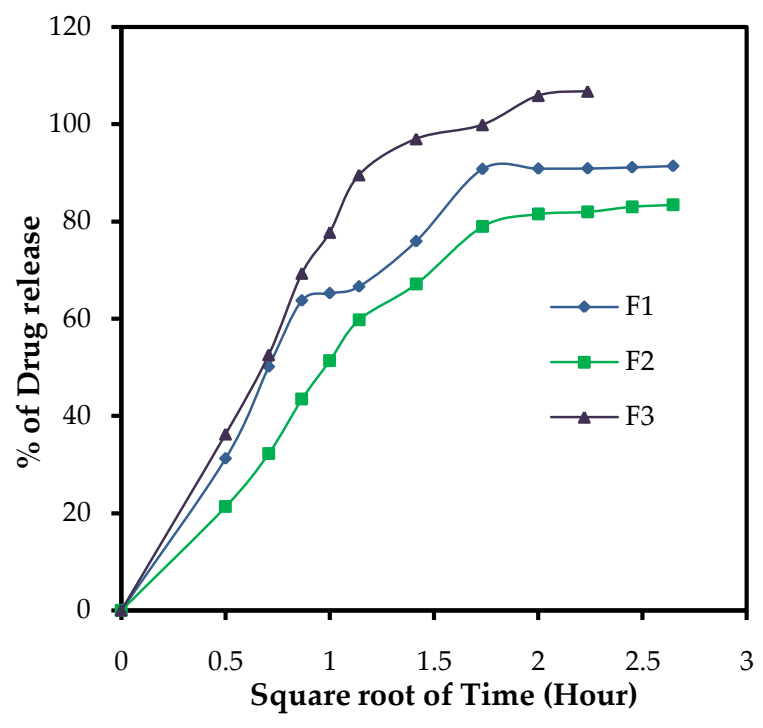

Figure 4: Higuchi plot of release kinetics of Carvedilol SR tablets of different formulations.

Microcrystalline Cellulose were used as release modifiers. To investigate the effect of these two release modifiers, the drug release data obtained were extrapolated by Zero order, First order, and Higuchi, Korsmeyer-Peppas and Hixson-Crowell equations (Higuchi, 1961; Ritger and Peppas, 1987; Korsmeyer et al., 1983). High correlation coefficient was observed in Higuchi plot (Table 3). Thus the drug release was proportional to the square root of time, indicating that the drug release is diffusion controlled. The diffusional exponent or $n$ values of the formulation F1, F2 and F3 were $0.28,0.39$ and 0.50 indicating Fickian type of release mechanism for formulation F1 and F2 and Non-Fickian (anomalous) type release mechanism (Table 3). The MDT values calculated were $1.40 \mathrm{hr}, 2.01 \mathrm{hr}$ and $0.65 \mathrm{hr}$ for formulation F1, F2 and F3 respectively.

\section{Effect of Release Modifiers on Carvedilol Release} Formulation F1 was modified by incorporating HPMC (100 mg) or Microcrystalline Cellulose (100 $\mathrm{mg}$ ), to develop new formulation F2 and F3 respectively, to observe the effect of release modifier on drug release. The tablets of formulation F1 containing only Kollidon SR polymer exhibited $91.42 \%$ release at 7 hours whereas tablets of formulation F2 (containing $100 \mathrm{mg}$ HPMC and $200 \mathrm{mg}$ Kollidon SR) and F3 (containing 100mg Microcrystalline Cellulose and $200 \mathrm{mg}$ Kollidon SR) exhibited $83.41 \%$ and 100 


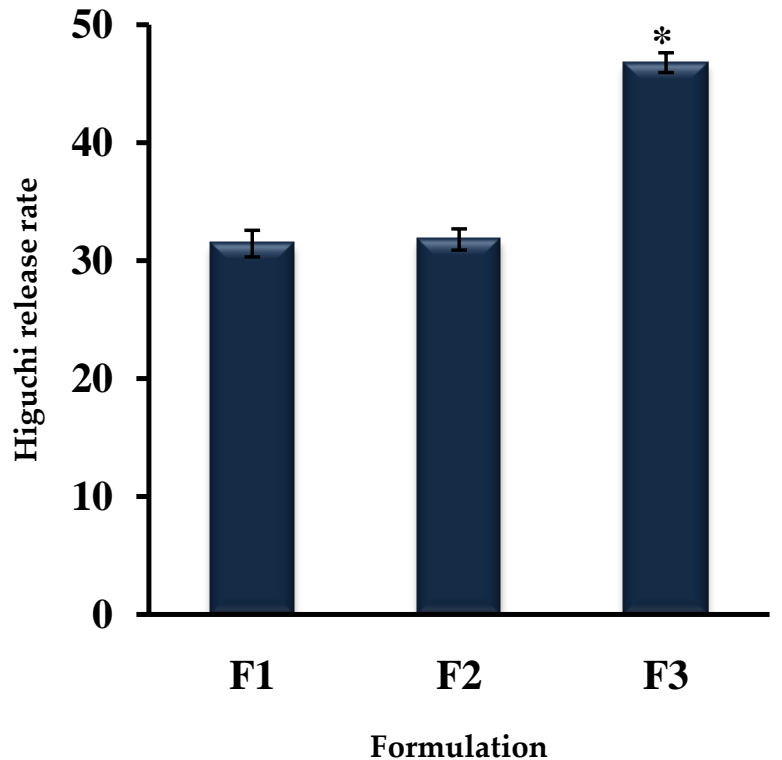

Figure 5: Bar diagram of Higuchi release rate of Carvedilol from different formulations.

* statistically significant ( $p<0.05$ vs F-1)

$\%$ release at time 7 and 4 hour respectively. Among these two release modifiers, HPMC containing tablets showed better release retarding capacity of carvedilol than another. The fact can be attributed to the hydrophilic nature of hydroxypropyl methylcellulose polymers. After salvation of the polymer chains, the dimensions of the polymer relaxation by stress of the penetrated solvent. This phenomenon is defined as swelling and it is characterized by the formation of a gel-like network surrounding the tablets (Alderman, 1987). This swelling and hydration property of HPMC causes an immediate formation of a surface barrier around the matrix tablet. The thickness of the barrier increases with time which governs the rate of drug release in swelling controlled pattern. The mechanical property of the surface hydrated gelatinous barrier play an important role in overall drug release (Talukdar et al., 1996). Microcrystalline cellulose (Avicel) containing tablets showed super imposable release pattern. The possible explanation is its superior drug carrying capacity and rapid disintegration.

\section{Higuchi Release Rate of Carvedilol from Different Formulations}

The slop of the Higuchi plot was calculated from their release rate. The formulation F3 containing Microcrystalline Cellulose showed the highest percentage of release $(46.77 \%)$. For formulation F1 and $\mathrm{F} 2$, the release rate found were $31.43 \%$ and $31.78 \%$, respectively.

\section{CONCLUSION}

Sustained release matrix tablets of Carvedilol were prepared using Kollidon SR as polymer and, HPMC and Microcrystalline Cellulose were used as release modifiers. The release rate of drug from the matrix tablets was significantly influenced by these two release modifiers. Formulation F1 containing Kollidon SR (300 mg) only and F2 (containing 100 mg HPMC and $200 \mathrm{mg}$ Kollidon SR) indicated that the release mechanism was dominated by Fickian Diffusion phenomenon whereas in case of formulation F3 (containing 100mg Microcrystalline Cellulose and $200 \mathrm{mg}$ Kollidon SR) increasing of release exponent $(\mathrm{n})$ indicated the shifting of release mechanism from Fickian to Non-Fickian direction. However, further investigation is required to establish in-vivo-in-vitro correlation to reveal the accurate pattern of drug release in vivo environment from these polymeric systems.

\section{ACKNOWLEDGEMENT}

The authors of the present study are thankful to Drug International Ltd. Dhaka, Bangladesh for providing, free of charge, Carvedilol for this study. The authors are also thankful to all the teachers and staffs of The Noakhali Science and Technology University for their support and cooperation.

\section{REFERENCES}

Alderman, D. A. (1987). A Review of cellulose ethers in hydrophilic matrices for oral controlled release dosage forms. Int J Pharm Tech Prod Mfr. 5: 1-9.

Beringer, P., Hoover, J. E. and Gelone, S. (2005). ExtendedRelease and Targeted Drug Delivery Systems. Remington: The Science and Practice of Pharmacy. 21 ${ }^{\text {st }}$ edition, Vol. 1, Lippincott Williams \& Wilkins, 939.

Billah, N., Yuen, K. H. and Peh., K. K. (1998). Diclofenac release from Eudragit containing matrice and effects of thermal treatment. Drug Dev. Ind. Pharm. 24: 45-50. [DOI]

Cardinal, J. R. (1984). Matrix systems. In: R. S. Langer and D. L. Wise, editors: Medical application of controlled release. $1^{\text {st }}$ edition. Boca Raton, Florida, CRC Press: 41-67. 
Higuchi, T. (1961). Mechanism of sustained action medication, Theoretical analysis of rate of release of solid drugs dispersed in solid matrices. J. Pharm. Sci. 52: 1145-1149. [DOI]

Korsmeyer, R. W., Gurny, R. and Doelker, E. M. (1983). Mechanism of solute release from porous hydrophilic polymers. Int. J. Pharm. 15: 25-35. [DOI]

Mockel, J. E. and Lippold, B. C. (1993). Zero order release from hydrocolloid matrices. Pharm Res, 10:1066-1070. [DOI]

Reja, M. S., Quadir, M. A. and Haider, S. S. (2003). Comparative evaluation of plastic, hydrophobic and hydrophilic polymers as matrices for controlled-release drug delivery, J Pharm Sci, 692: 274-291.
Ritger, P. L. and Peppas, N. A. (1987). A simple equation for description of solute release II. Fickian and anomalous release from swellable devices. J Control Rel, 5: 37-42. [DOI]

Shato, H., Miyagawa, Y. and Okabe, T. (1997). Dissolution mechanism of diclofenac sodium from wax matrix granules. J Pharm Sci, 86(8): 929-934. [DOI]

Talukdar, M. M., Michoel, A. and Rhombaut. (1996).Comparative study on Xanthan gum and hydroxypropyl methylcellulose as matrices for controlled-release drug delivery I. Compaction and in vitro drug release behavior. Int. J .Pharm.129: 231-241. [DOI] 